\title{
Prevalencia Serológica de Leptospira spp. en Cerdos Silvestres (Sus scrofa) en Bahía de Samborombón, provincia de Buenos Aires, República Argentina, en el Periodo 2013-2015
}

\author{
Petrakovsky Jessica $^{1 *}$, Carpinetti Bruno ${ }^{2}$, Antonuci Andrea ${ }^{1}$ \\ ${ }^{1}$ Laboratorio de Referencia de la O.I.E. en Leptospirosis. Dirección de Laboratorios y Control Técnico. Servicio Nacional de Sanidad y Calidad \\ Agroalimentaria (SENASA). Buenos Aires, Argentina. \\ ${ }^{2}$ Gestión Ambiental/Ecología - Instituto de Ciencias Sociales y Administración - Universidad Nacional Arturo Jauretche. Buenos Aires, Argentina \\ *jpetrako@senasa.gob.ar
}

\begin{abstract}
RESUMEN
Los animales silvestres en su medio natural o en condiciones de cautividad son componentes vitales en el ciclo epidemiológico de enfermedades zoonóticas, entre las cuales se encuentra leptospirosis, actuando como reservorios, hospedadores de mantenimiento, portadores u hospedadores accidentales, participando en la transmisión de diferentes serovares patógenos. Objetivo: detectar la presencia de anticuerpos contra Leptospira spp. en jabalíes (Sus scrofa) y cerdos asilvestrados (Sus scrofa domestica) en estado silvestre que no presentaban sintomatología clínica, provenientes de Bahia Samborombón, Buenos Aires. Materiales y métodos: la población bajo estudio comprendió una muestra no representativa de la de la población porcina silvestre (Sus scrofa) recolectada en el periodo 2013-2015. Los sueros fueron analizados por la técnica de MAT (prueba de microaglutinación). Resultados: el 34,5\% de las muestras resultaron positivas a la prueba tamiz empleada (primera etapa del MAT), siendo esta serorreactividad positiva exclusivamente al serovar Pomona. Al realizar la titulación de los sueros (segunda etapa del MAT), el porcentaje de positividad se mantuvo en el mismo valor. El 77 \% de las muestras presentó un título final menor a 1:200. Conclusión: la existencia de sueros positivos indica el contacto de los animales con la leptospira. Considerando que no es posible erradicar la leptospirosis debido a que se mantiene en sus reservorios silvestres, contar con datos epidemiológicos en la región permitirá implementar medidas de prevención y control de la enfermedad.
\end{abstract}

\section{Serological Prevalence of Leptospira spp. in Wild Swine (Sus scrofa) in Bahía de Samborombón, Buenos Aires province, Argentina, in 2013-2015}

\footnotetext{
ABSTRACT

Wild animals in their natural environment or in captivity are vital components in the epidemiological cycle of zoonotic diseases, including leptospirosis, acting as reservoirs, hosts maintenance carriers or accidental hosts, participating in the transmission of different pathogen serovars. Objective: To detect the presence of antibodies against Leptospira spp. in wild boars (Sus scrofa) and feral pigs (Sus scrofa domestica), in the wild, who had no clinical symptoms, from Bahia de Samborombón, Buenos Aires. Materials and methods: the population under study included a non-representative sample of the population of wild swine (Sus scrofa) collected in the period 2013-2015. Sera were analyzed by MAT (microscopic agglutination test). Results: $34.5 \%$ of the samples tested were positive for the screening test used (first stage of the MAT) and all of them reacted only to serovar Pomona. When the sera titration was performed (second stage of the MAT), the percentage of positivity remained in the same value. Seventy seven percent of the samples showed a final titer lower than 1:200. Conclution: the existence of positive sera indicates the contact of the animals with Leptospira.

Whereas it is not possible to eradicate leptospirosis because it keeps in its wild reservoirs, dispose of epidemiological data in the region will allow taking actions to the prevention and control of this disease
} 


\section{INTRODUCCIÓN}

Las zoonosis son enfermedades de los animales vertebrados que se transmiten naturalmente al hombre. De los 1.415 patógenos humanos conocidos en el mundo, $61 \%$ son zoonóticos y por lo tanto tienen relación directa con las actividades de la Salud Pública Veterinaria. (OPS, 2015)

Varios agentes zoonóticos están presentes en cerdos. La trichinelosis es una zoonosis ampliamente distribuida por todo el mundo y afecta tanto a animales domésticos como salvajes de todos los continentes. En Argentina se ha identificado Trichinella spiralis en armadillos, jabalíes y pumas (Bono Battistoni, 2015). Su infección es endémica y los casos humanos han ido en aumento (Ribicich, 2005).

Otros agentes zoonóticos presentes en cerdos son: Salmonella spp., Escherichia coli, Campylobacter spp., Yersinia enterocolítica, Cryptosporidium parvum, Giardia intestinalis, Balantidium coli (responsables de infección gastrointestinal), Microsporum nanum, Microsporum canis, Trichophyton mentagrophytes y $\mathrm{T}$. verrucosum (responsables de infecciones micóticas), virus de la influenza, virus rábico, Hepatitis-E, Streptococcus suis, Erysipela rhusiopathiae, Brucella suis y Trichuris suis (Cornell, 2014)

La leptospirosis es la zoonosis de difusión más amplia en todo el mundo; está presente en todos los continentes excepto la Antártida y se ha encontrado evidencia de portadores de Leptospira en prácticamente todas las especies mamíferas examinadas. Los seres humanos se infectan comúnmente a través del contacto ocupacional, recreativo o doméstico con la orina de animales portadores, ya sea directamente o a través de agua contaminada o en el suelo (Romero, 2011).

Numerosos animales silvestres son portadores y vectores de leptospiras, siendo los roedores los principales reservorios para estos agentes infecciosos que han evolucionado o coevolucionado con el huésped, adaptándose perfectamente a algunas especies de la fauna silvestre que no sufren la enfermedad ni mueren por la infección, por lo que pasan a ser reales e importantes huéspedes de mantención de este patógeno virulento, prácticamente de por vida. En consecuencia, se transforman en fuente de infección para la especie humana y también para animales domésticos, siendo a veces la causa de la aparición de enfermedades emergentes o bien de reemergentes, de ahí que sea muy importante el conocimiento geográfico de los nichos ecológicos de los reservorios (Zamora, 1999).

Además de la amplia distribución universal, es una de las enfermedades con mayor impacto económico por las grandes pérdidas que ocasiona en la ganadería (abortos, mortalidad perinatal, disminución de la producción láctea, etc.) (AAVLD, 1994).

En veterinaria, el cerdo es el mejor ejemplo de portador/excretor, ya que una vez localizadas las leptospiras en el riñón, este animal se transforma en excretor de por vida, jugando un rol de gran importancia en el ciclo de la leptospirosis, semejante al roedor. No ocurre lo mismo con las otras especies, el bovino entre ellas, cuya capacidad excretora, de duración variable, no es permanente (AAVLD, 1994).

Se ha demostrado que existe un contacto de los cerdos domésticos con la leptospira a lo largo de toda la República Argentina. El 30\% de los animales muestreados en el año 2006 resultaron positivos a nivel nacional y a nivel provincial se observó una tendencia similar. El serovar prevalente fue Castellonis, seguido por Icterohaemorrhagiae y Pomona. Existe un agrupamiento de los animales reaccionantes en la región Central y Norte del País, el cual coincide geográficamente con las áreas donde se encuentran mayor cantidad de explotaciones porcinas. (Petrakovsky, 2012)

En Argentina, algunas de las poblaciones silvestres de Sus scrofa son descendientes de las diversas razas del cerdo doméstico (Sus scrofa domestica) lanzado desde la colonización española. Además, deliberadamente se introdujo puro jabalí euroasiático alrededor de 1906 para fines de caza deportiva. La hibridación entre las poblaciones silvestres y domésticas también es común, lo que resulta en un patrón de distribución muy confuso y la interrelación de formas naturalizadas, domésticas e híbridas. Actualmente, la especie se distribuye ampliamente en regiones donde los hábitats disponibles son menos afectados por las actividades antropogénicas (Merino, 2003).

La prueba de Microaglutinación (MAT) en la que 
se emplean antígenos vivos es la prueba serológica recomendada para el diagnóstico de leptospirosis en el "Código Sanitario para los Animales Terrestres" de la Organización Mundial de Sanidad Animal. Constituye la prueba de referencia frente a la que se evalúan todas las otras pruebas serológicas (OIE, 2003).

\section{MATERIALES Y METODOS \\ Tipo de estudio}

Se realizó un muestreo pasivo no aleatorio. La población bajo estudio comprendió una muestra no representativa de la de la población porcina silvestre (Sus scrofa). Dada la dificultad de obtener la muestra por ser animales salvajes, los resultados obtenidos no permitirán estimar valores de prevalencia reales, sino que brindarán información sobre la presencia de anticuerpos antileptospiras en los animales muestreados.

\section{Sitio de estudio}

El muestreo fue realizado por personal de la Dirección de Sanidad Animal del SENASA en la Bahía de Samborombón (provincia de Buenos Aires, República Argentina).

El período de estudio estuvo comprendido entre enero de 2013 y mayo 2015.

\section{Obtención de las Muestras}

La captura de los animales estuvo a cargo de la Dirección Nacional de Sanidad Animal en el marco de un proyecto cuyo objetivo fue evaluar la presencia y distribución de enfermedades de los porcinos con especial énfasis en enfermedades zoonóticas, de importancia para la producción animal y para la conservación de especies amenazadas o en peligro de la fauna silvestre, en las poblaciones de cerdos ferales y jabalíes de la Argentina. Los animales fueron capturados y necropsiados en el acto extrayéndose las muestras necesarias para el diagnóstico de las enfermedades mencionadas. Cabe aclarar que la toma de muestras de una especie exótica altamente perjudicial para la conservación y la importancia que conlleva este proyecto para la salud pública, y la producción ganadera se encuadran dentro de las previsiones de la ley en las distintas jurisdicciones en las que se pretende muestrear (Carpinetti, 2014). Las muestras de suero fueron remitidas inmediatamente al laboratorio en las condiciones recomendadas en el Capitulo 1.1.1 del "Manual de las Pruebas de Diagnóstico y de las Vacunas para los Animales Terrestres" (OIE, 2014).

Se obtuvo un total de 90 sueros.

\section{Análisis de los sueros}

Las muestras obtenidas fueron analizadas mediante la prueba de MAT (prueba de microaglutinación).

El procedimiento utilizado en la prueba de microaglutinación (MAT) es el descripto en el "Manual de las Pruebas de Diagnóstico y de las Vacunas para los Animales Terrestres. OIE" (2014). Se utilizó una batería de ocho serovariedades de Leptospira sp. (Canicola, Castellonis, Grippothyphosa, Icterohaemorragiae, Pomona, Pyrogenes, Tarassovi y Wolffi) que en estudios anteriores mostraron estar presentes en Argentina (AAVLD, 1994).

El criterio de positividad fue un título mayor o igual a 1:100. En una primera etapa se procesaron los sueros diluidos 1:100 para identificar los positivos, los cuales fueron titulados posteriormente para determinar el serovar predominante en la reacción con el suero y el valor de su título.

\section{RESULTADOS}

De los 90 animales muestreados, 31 resultaron positivos, es decir, el $34,5 \%$ de las muestras resultaron positivas a la dilución 1:100 en la primera etapa del MAT (tamizaje). En la titulación final se observó que se mantuvo el porcentaje de positividad (34.5\%). (Cuadro 1)

Los sueros analizados reaccionaron positivamente al serovar Pomona sin presentar reacciones cruzadas con los demás serovares.

De los 31 sueros positivos el $77.5 \%$ presentó títulos menores o iguales a 1:200, mientras que en el $32.5 \%$ restante se observaron títulos comprendidos entre 1:400 y 1:1600. (Tabla 1). 
Cuadro 1. Cantidad de animales positivos y negativos y sus respectivos porcentajes.

\begin{tabular}{|c|c|c|c|}
\hline & Positivos & Negativos & Total \\
\hline $\begin{array}{c}\text { Animales } \\
\text { muestreados }\end{array}$ & 31 & 59 & 90 \\
\hline Porcentaje & 34.5 & 65.5 & 100 \\
\hline
\end{tabular}

Tabla 1. Frecuencia de aparición de los títulos finales positivos a serovar Pomona (iguales o mayores a 1:100) expresados en números absolutos y porcentajes.

\begin{tabular}{|c|c|c|c|c|c|c|}
\hline $\begin{array}{c}\text { Dilución } \\
\text { de suero }\end{array}$ & $1: 100$ & $1: 200$ & $1: 400$ & $1: 800$ & $1: 1600$ & Total \\
\hline $\begin{array}{c}\text { Canti- } \\
\text { dad }\end{array}$ & 15 & 9 & 4 & 2 & 1 & 31 \\
\hline $\begin{array}{c}\text { Porcen- } \\
\text { tajes }\end{array}$ & 48.5 & 29 & 13 & 6.5 & 3 & 100 \\
\hline
\end{tabular}

\section{DISCUSIÓN}

El 34,5\% de los animales muestreados resultaron positivos siendo exclusivamente el serovar reaccionante Pomona, ya que no se evidenciaron reacciones cruzadas con los otros serovares utilizados en la prueba tamiz del MAT.

Al momento de la realización de este trabajo no se hallaron estudios relacionados con la vigilancia de leptospirosis en porcinos silvestres en Argentina.

Cabe mencionar que se analizaron muestras de cerdos silvestres (Sus Scrofa) provenientes de otras provincias en este mismo periodo las cuales también mostraron serorreactividad positiva frente al serovar Pomona ( 3 positivas de 11 en Misiones y 2 positivas de 8 en Río Negro).

$\mathrm{Si}$ bien no se han publicado datos sobre aislamientos de leptospiras en esta especie, a partir de los resultados obtenidos se podría inferir que ejemplares de Sus Scrofa estarían en contacto con el serovar Pomona en varias provincias del país.

La información remitida en el presente trabajo constituye un primer paso en el estudio de la prevalencia serológica de leptospirosis en porcinos silvestres y debería ser ampliado con información relativa a otras áreas de nuestro país.

El monitoreo de la prevalencia serológica puede ser complementado utilizando técnicas diagnóstico orientadas a la detección del patógeno tales como el aislamiento bacteriológico, mediante el cultivo en medios especiales para Leptospira spp. y pruebas moleculares. El uso de las mencionadas técnicas sería un aporte fundamental para determinar la infección de los animales con Leptospira spp., la excreción de la bacteria y el rol de estos animales como portador de la misma.

Es fundamental contar con datos epidemiológicos en la región para poder implementar medidas de prevención y control adecuadas, ya que la leptospirosis es una enfermedad de muy difícil erradicación debido a que se mantiene en sus reservorios silvestres.

\section{CONCLUSIONES}

La existencia de anticuerpos en los sueros de los animales muestreados indica que existe un contacto de los cerdos silvestres (Sus Scrofa) con la leptospira en la Bahía de Samborombón.

Dado el importante rol de los porcinos como reservorios en la cadena epidemiológica de leptospirosis estos hallazgos ponen de manifiesto la relevancia de continuar estudios de vigilancia epidemiológica sobre la población de estos animales y otros mamíferos silvestres.

\section{LITERATURA CITADA}

Adler, B., \& Moctezuma, A. (2010). Leptospira y Leptospirosis. Veterinary Microbiology, 140, pp. 287-296.

Asociación Argentina de Veterinarios de Laboratorios de Diagnóstico. (1994) Manual de Leptospirosis.

Bono Battistoni, M.F., Marengo R., Orcellet, E., Peralta, J.L. (2015). Determinación de Trichinella spp. en jabalí (Sus scrofa). Revista Veterinaria Argentina, vol. XXXII, $n^{\circ} 321$.

Carpinetti, B., Castresana, G. et al. (2014 juliodiciembre). Vigilancia Epidemiológica en Poblaciones de Cerdos Silvestres (Sus scrofa). Revista SNS, N5-6.

Cornell University: Potential Zoonoses Associated with Swine. 2014. Disponible en: https://ras. research.cornell.edu/care/documents/OHS/ zoonosis_information_sheet_swine.pdf (visto el $23 / 11 / 15)$ 
Merino, M. \& Carpinetti, B. (2003 julio-diciembre) Feral pig (Sus scrofa) population. Estimates in Bahía Samborombón Conservation Area, Buenos Aires Province, Argentina. Mastozoología Neotropical, 10, $\mathrm{N}^{\circ} 2$, pp. 269-275.

Organización Mundial de la Salud, Organización Panamericana de la Salud. Temas de salud: zoonosis. 2015. Disponible en http://www. paho.org/hq/index.php? option $=$ com 